\title{
Passive Noise Control Strategies for Jets Exhausting over Flat Surfaces: an LES Study
}

\author{
Colby Horner ${ }^{* 1}$, Adrian Sescu $^{\dagger 1}$, Mohammed Afsar ${ }^{\ddagger 2}$, Eric Collins ${ }^{\S 3}$, and Mahdi \\ Azarpeyvand $\boldsymbol{\top 4}$ \\ ${ }^{1}$ Department of Aerospace Engineering, Mississippi State University, MS 39762 \\ ${ }^{2}$ Department of Mechanical \& Aerospace Engineering, Strathclyde University, Glasgow, UK \\ ${ }^{3}$ Center for Advanced Vehicular Systems, Mississippi State University, MS 39762 \\ ${ }^{4}$ Department of Mechanical Engineering, University of Bristol, Bristol, UK
}

\begin{abstract}
Unconventional aircraft propulsion configurations have to be considered in the future to address environmental issues, including air traffic noise that is know to affect communities surrounding airports. One approach involves rectangular jets in the vicinity of flat surfaces that are parallel to the jet axis, but previous experimental work indicated that there is an increase in the noise generated by these configurations, mainly associated with the effect that the plate trailing edge exerts on the flow. In this work, we employ large eddy simulations to investigate the potential of wall deformations at the trailing edge to reduce jet noise. We consider a high aspect ratio rectangular nozzle exhausting a jet over a flat surface in different configurations, and estimate the farfield noise using the FFowcs Williams and Hawkins acoustic analogy.
\end{abstract}

\section{Introduction}

There are certain aircraft propulsion configurations, for which high-speed jets are emitted over wing surfaces generating significant flow distortions that can contribute to the increase of the overall noise. One example is the aircraft engine placed on top of the wing in an attempt to shield the jet noise that would propagate to the ground, and at the same time maxime aircraft performance. Another example is an aircraft carrier deck supporting military aircraft with jets interacting with the surface during take-off and landing operations. Besides increased farfield noise, there are also structural concerns from this interactions since the intense flow and noise propagating downstream or upstream can compromise the airframe structural integrity.

Jet-surface interaction noise is usually associated with an increase in low frequency noise as a result of the interaction between a turbulent jet and a flat surface that is parallel to the jet axis. This jet noise increase is generally the result of two sources: the first source is called scrubbing noise, and the second is the trailing edge scattering noise (Brown and Wernet ${ }^{7}$ ). Scrubbing is generated by the interaction of the turbulent flow with the flat surface, while the trailing edge noise, which is the dominant noise source $\left(\right.$ Brown, ${ }^{6}$ Podboy ${ }^{11}$ ), is produced when the turbulent flow interacts with the edge of the plate and transitions to a mixing layer. There is also an additional noise component that is affected by the jet surface interaction, which is associated with the turbulent mixing either upstream or downstream of the trailing edge.

Interactions between jet flows and flat surfaces that are installed just downstream of the nozzle exit have been studied in the last decades. For example, Seiner and Manning ${ }^{12}$ considered the interaction between a supersonic jet and a flat plate from a rectangular nozzle; they showed that the distance between the nozzle

\footnotetext{
*Research Assistant, Department of Aerospace Engineering, Mississippi State University, MS 39762; member AIAA.

${ }^{\dagger}$ Associate Professor, Department of Aerospace Engineering, Mississippi State University, MS 39762; Associate Fellow AIAA.

${ }^{\ddagger}$ Chancellor Fellow, Department of Mechanical \& Aerospace Engineering, Strathclyde University, 75 Montrose St. Glasgow, G1 1XJ, UK; AIAA member.

$\S$ Research Assistant Professor, Center for Advanced Vehicular Systems, Mississippi State University, MS 39762; member AIAA.

`Professor, Department of Mechanical Engineering, University of Bristol, University Walk, Clifton BS8 1TR, UK.
} 
exit and flat surface was an important parameter which can impact the screech noise. Bridges ${ }^{5}$ and Zaman et al., ${ }^{15}$ as part of a series of extensive studies conducted at NASA Glenn Research Center, found that the length of the plate has an effect on jet noise characteristics. Ibrahim et al. ${ }^{9}$ studied the effect of turbulence characteristics of jet flows on the radiated jet noise. Most recently, Behrouzi and McGuirk ${ }^{2}$ carried out an experimental study of an underexpanded rectangular supersonic jet flow with a very high aspect ratio. They showed that the presence of an aft-deck at the nozzle exit had a strong effect on jet development and it reduced turbulence levels in the near field.

Our main objective of this work is to investigate whether surface deformations or different types of actuation in proximity of the trailing edge can potentially reduce the jet noise. To this end, we perform a suite of large eddy simulations of different geometrical and flow conditions, and evaluate the farfield noise radiation using FFowcs Williams and Hawkins acoustic analogy method. The numerical tool is a highaccurate solver, discretizing the unsteady, compressible, conservative form of the Navier-Stokes equations written in body-fitted curvilinear coordinates. We consider a high aspect ratio rectangular jet, which allows us to simplify the geometry by considering a spanwise slice of width $2 h$ ( $h$ is the height of the nozzle). In section II, the governing equations, the scalings and the numerical algorithm are succinctly described. In section III, several preliminary results are presented and discussed.

\section{Problem formulation and numerical algorithm}

\section{A. Scalings}

The equations considered here involve a generalized curvilinear coordinate transformation, which is written in the three-dimensional form as $\xi=\xi(x, y, z), \eta=\eta(x, y, z), \zeta=\zeta(x, y, z)$, where $\xi, \eta$ and $\zeta$ are the spatial coordinates in the computational space corresponding to the streamwise, wall-normal and spanwise directions, and $x, y$ and $z$ are the spatial coordinates in physical space. This transformation allows for a seamless mapping of the solution from the computational to the physical space and vice-versa. All dimensional spatial coordinates are normalized by the reference length $D$ associated with the fractal geometry,

$$
(x, y, z)=\frac{\left(x^{*}, y^{*}, z^{*}\right)}{D},
$$

the velocity is scaled by the freestream velocity magnitude $V_{\infty}^{*}$,

$$
(u, v, w)=\frac{\left(u^{*}, v^{*}, w^{*}\right)}{V_{\infty}^{*}},
$$

the pressure by the dynamics pressure at infinity, $\rho_{\infty}^{*} V_{\infty}^{* 2}$, and temperature by the freestream temperature, $T_{\infty}^{*}$. Reynolds number based on the spanwise separation, Mach number and Prandtl number are defined as

$$
R_{\lambda}=\frac{\rho_{\infty}^{*} V_{\infty}^{*} D}{\mu_{\infty}^{*}}, \quad M a=\frac{V_{\infty}^{*}}{a_{\infty}^{*}}, \quad \operatorname{Pr}=\frac{\mu_{\infty}^{*} C_{p}}{k_{\infty}^{*}}
$$

where $\mu_{\infty}^{*}, a_{\infty}^{*}$ and $k_{\infty}^{*}$ are freestream dynamic viscosity, speed of sound and thermal conductivity, respectively, and $C_{p}$ is the specific heat at constant pressure. All simulations are performed for air as an ideal gas.

\section{B. Governing equations}

In conservative form, the filtered Navier-Stokes equations are written as

$$
\mathbf{Q}_{t}+\mathbf{F}_{\xi}+\mathbf{G}_{\eta}+\mathbf{H}_{\zeta}=\mathbf{S} .
$$

where subscript denote derivatives, the vector of conservative variables is given by

$$
\mathbf{Q}=\frac{1}{J}\left\{\rho, \quad \rho u_{i}, \quad E\right\}^{T}, i=1,2,3
$$


$\rho$ is the non-dimensional density of the fluid, $u_{i}=(u, v, w)$ is the non-dimensional velocity vector in physical space, and $E$ is the total energy. The flux vectors, $\mathbf{F}, \mathbf{G}$ and $\mathbf{H}$, are given by

$$
\begin{aligned}
& \mathbf{F}=\frac{1}{J}\left\{\rho U, \quad \rho u_{i} U+\xi_{x_{i}}\left(p+\tau_{i 1}\right), \quad E U+p \tilde{U}+\xi_{x_{i}} \Theta_{i}\right\}^{T}, \\
& \mathbf{G}=\frac{1}{J}\left\{\rho V, \quad \rho u_{i} V+\eta_{x_{i}}\left(p+\tau_{i 2}\right), \quad E V+p \tilde{V}+\eta_{x_{i}} \Theta_{i}\right\}^{T}, \\
& \mathbf{H}=\frac{1}{J}\left\{\rho W, \quad \rho u_{i} W+\zeta_{x_{i}}\left(p+\tau_{i 3}\right), \quad E W+p \tilde{W}+\zeta_{x_{i}} \Theta_{i}\right\}^{T}
\end{aligned}
$$

where the contravariant velocity components are given by

$$
U=\xi_{x_{i}} u_{i}, \quad V=\eta_{x_{i}} u_{i}, \quad W=\zeta_{x_{i}} u_{i}
$$

with the Einstein summation convention applied over $i=1,2,3$, the shear stress tensor and the heat flux are given as

$$
\begin{gathered}
\tau_{i j}=\frac{\mu}{R e}\left[\left(\frac{\partial \xi_{k}}{\partial x_{j}} \frac{\partial u_{i}}{\partial \xi_{k}}+\frac{\partial \xi_{k}}{\partial x_{i}} \frac{\partial u_{j}}{\partial \xi_{k}}\right)-\frac{2}{3} \delta_{i j} \frac{\partial \xi_{l}}{\partial x_{k}} \frac{\partial u_{k}}{\partial \xi_{l}}\right] \\
\Theta_{i}=u_{j} \tau_{i j}+\frac{\mu}{(\gamma-1) M_{\infty}^{2} \operatorname{RePr}} \frac{\partial \xi_{l}}{\partial x_{i}} \frac{\partial T}{\partial \xi_{l}}
\end{gathered}
$$

respectively, and $\mathbf{S}$ is the source vector term.

The pressure $p$, the temperature $T$ and the density of the fluid are combined in the equation of state, $p=$ $\rho T / \gamma M_{\infty}^{2}$ when non-chemically-reacting flows are considered; when chemically reacting flows are taken into account, equations of species are used instead. Other notations include the dynamic viscosity $\mu$, Reynold's number $R e=\rho V_{\infty}^{*} \lambda^{*} / \mu$ based on a characteristic velocity $V_{\infty}^{*}$, and a characteristic length $L$, the free-stream Mach number $M_{\infty}=V_{\infty}^{*} / a_{\infty}^{*}$. The Jacobian of the curvilinear transformation from the physical space to computational space is denoted by $J$. The derivatives $\xi_{x}, \xi_{y}, \xi_{z}, \eta_{x}, \eta_{y}, \eta_{z}, \zeta_{x}, \zeta_{y}$, and $\zeta_{z}$ represent grid metrics. The dynamic viscosity and thermal conductivity $k$ is linked to the temperature using the Sutherland's equations in dimensionless form,

$$
\mu=T^{3 / 2} \frac{1+C_{1} / T_{\infty}}{T+C_{1} / T_{\infty}} ; \quad k=T^{3 / 2} \frac{1+C_{2} / T_{\infty}}{T+C_{2} / T_{\infty}}
$$

where for air at sea level, $C_{1}=110.4 K, C_{2}=194 K$, and $T_{\infty}$ is a reference temperature. There are no subgrid scale terms in equation (4) since an implicit large eddy simulation framework is considered here.

\section{Numerical framework}

The compressible Navier-Stokes equations are solved in the framework of implicit large eddy simulations, where numerical filtering is applied to account for the missing sub-grid scale energy. The numerical algorithm uses high-order finite difference approximations for the spatial derivatives and explicit time marching. The time integration is performed using a third order TVD Runge-Kutta method (Shu and Osher ${ }^{13}$ ) written in the form

$$
\begin{aligned}
\mathbf{Q}^{(0)} & =\mathbf{Q}^{n} \\
\mathbf{Q}^{(1)} & =\mathbf{Q}^{(0)}+\Delta t L\left(u^{(0)}\right) \\
\mathbf{Q}^{(2)} & =\frac{3}{4} \mathbf{Q}^{(0)}+\frac{1}{4} \mathbf{Q}^{(1)}+\frac{1}{4} \Delta t L\left(\mathbf{Q}^{(1)}\right) \\
\mathbf{Q}^{n+1} & =\frac{1}{3} \mathbf{Q}^{(0)}+\frac{2}{3} \mathbf{Q}^{(1)}+\frac{2}{3} \Delta t L\left(\mathbf{Q}^{(2)}\right)
\end{aligned}
$$

where $L(\mathbf{Q})$ is the residual. 
The spatial derivatives are discretized using dispersion-relation-preserving schemes of Tam and Webb ${ }^{14}$ or a high-resolution 9-point dispersion-relation-preserving optimized scheme of Bogey et al. ${ }^{3}$ The first derivative at the $l$ th node is approximated using $M$ values of $f$ to the right and $N$ values of $f$ to left of the node.

$$
\left(\frac{\partial f}{\partial x}\right)_{l} \simeq \frac{1}{\Delta x} \sum_{j=-N}^{M} a_{j} f_{l+j}
$$

By taking the Fourier transform of the above equation, the coefficients $a_{j}$ are found by minimizing the integrated error of the difference between the wavenumber of the finite difference scheme and the wavenumber of the Fourier transform of the finite difference scheme. The coefficients $a_{j}$ are given in table 1.

\begin{tabular}{|r|r|r|r|r|}
\hline Stencil & $a_{1}=-a_{-1}$ & $a_{2}=-a_{-2}$ & $a_{3}=-a_{-3}$ & $a_{4}=-a_{-4}$ \\
\hline$D R P$ & 0.77088238 & -0.16670590 & 0.02084314 & 0 \\
$F D o 9 p$ & 0.84157012 & -0.24467863 & 0.05946358 & -0.00765090 \\
\hline
\end{tabular}

Table 1: Weights of the centered stencils

To damp out the unwanted high wavenumber waves from the solution, high-order spatial filters, as developed by Kennedy and Carpenter, ${ }^{10}$ are used. No slip boundary condition for velocity and adiabatic condition for temperature are imposed at the solid surface. Sponge layers are imposed in the proximity of the far-field boundaries, regions that are outside the flow domain of interest (this is combined with grid stretching and sponge layers to damp the unwanted waves). These sponge layers are designed to damp out the waves of all wavenumbers leaving the domain or reflecting back from the far-field boundaries.

Shock capturing techniques are needed to avoid unwanted oscillations that may propagate from potential discontinuities that can develop in supersonic flows. We apply a shock capturing methodology that was proven to work efficiently for high-order, nonlinear computations (Bogey et al. ${ }^{4}$ ). Since in the present work high-order, central-difference schemes are used to achieve increased resolution of the propagating disturbances, a straightforward approach is a model which introduces sufficient numerical viscosity in the area of the discontinuities, and negligible artificial viscosity in the rest of the domain. A shock-capturing technique, suitable for simulations involving central differences in space is applied, based on the general explicit filtering framework. The technique introduces selective filtering at each grid vertex to minimize numerical oscillations, and shock-capturing in the areas where discontinuities are present (more details can be found in Bogey et al. ${ }^{4}$ ).

The mean flow used to initialize the LES is obtained from RANS simulations, where a classical $k-\epsilon$ turbulence model is applied to account for fluctuations. 


\section{Preliminary Results}

Preliminary results are presented and discussed for a high aspect ratio rectangular jet exhausting over a flat surface located underneath the jet flow. Because the aspect ratio of the nozzle exit is high, we simplify the problem by considering only a portion from the nozzle along the spanwise direction, in the assumption that the flow is statistically two-dimensional (this assumption was also considered by Zaman et al. ${ }^{16}$ ). Thus, the spanwise length of the flow domain is set to twice the height of the nozzle, and periodic boundary conditions are imposed in the lateral direction. The flat surface is parallel to the jet axis and located underneath the nozzle at one height from the center of the nozzle, while the leading edge is located at five nozzle heights downstream of the nozzle exit. The geometry of the nozzle and the flat plate with deformed trailing edge are shown in figure 1, where the deformations resembles a sine function in the spanwise direction and a tangent hyperbolic function in the streamwise direction. The Reynolds number based on the jet velocity and the height of the nozzle is 500,000, and the acoustic Mach number is 0.8.

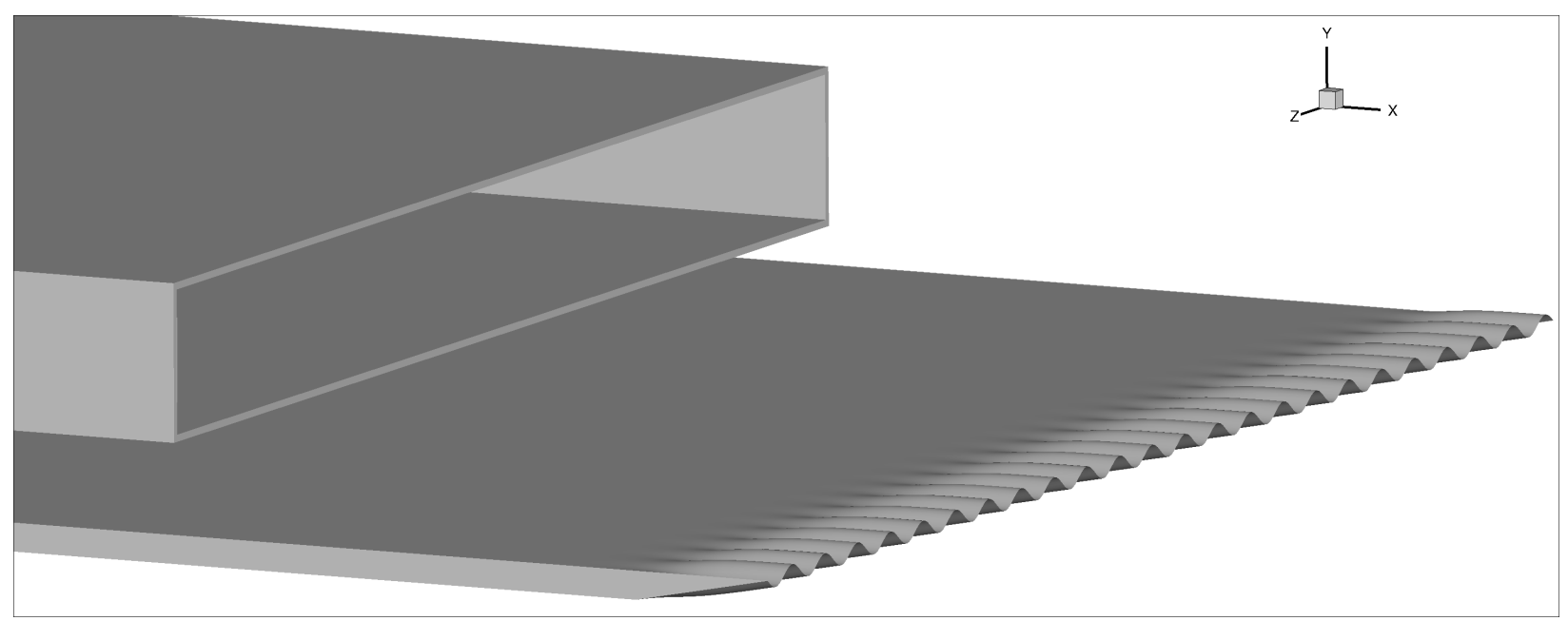

Figure 1: Rectangular nozzle and the flat surface underneath it.

The mean flow, which is used to initialize the LES, is obtained from a separate two-dimensional RANS simulation using a $\kappa-\epsilon$ turbulence model. Three temperature ratios $T R=T_{j} / T_{\infty}$ (where $T_{j}$ is the temperature of the jet, and $T_{\infty}$ is the ambient temperature) were simulated, with RANS results shown in figures 2-6. Contours of velocity magnitude that are plotted in figure 2 indicate, as expected, that the length of the potential core decreases as the temperature ratio $T R$ increases from 1 to 3 . At the same time, the turbulent kinetic energy levels increase as TR increases as noticed from figure 3. There is a slight deviation of the jet axis from the horizontal direction because of the presence of the surface, which creates a slight pressure gradient in the wall-normal direction. 


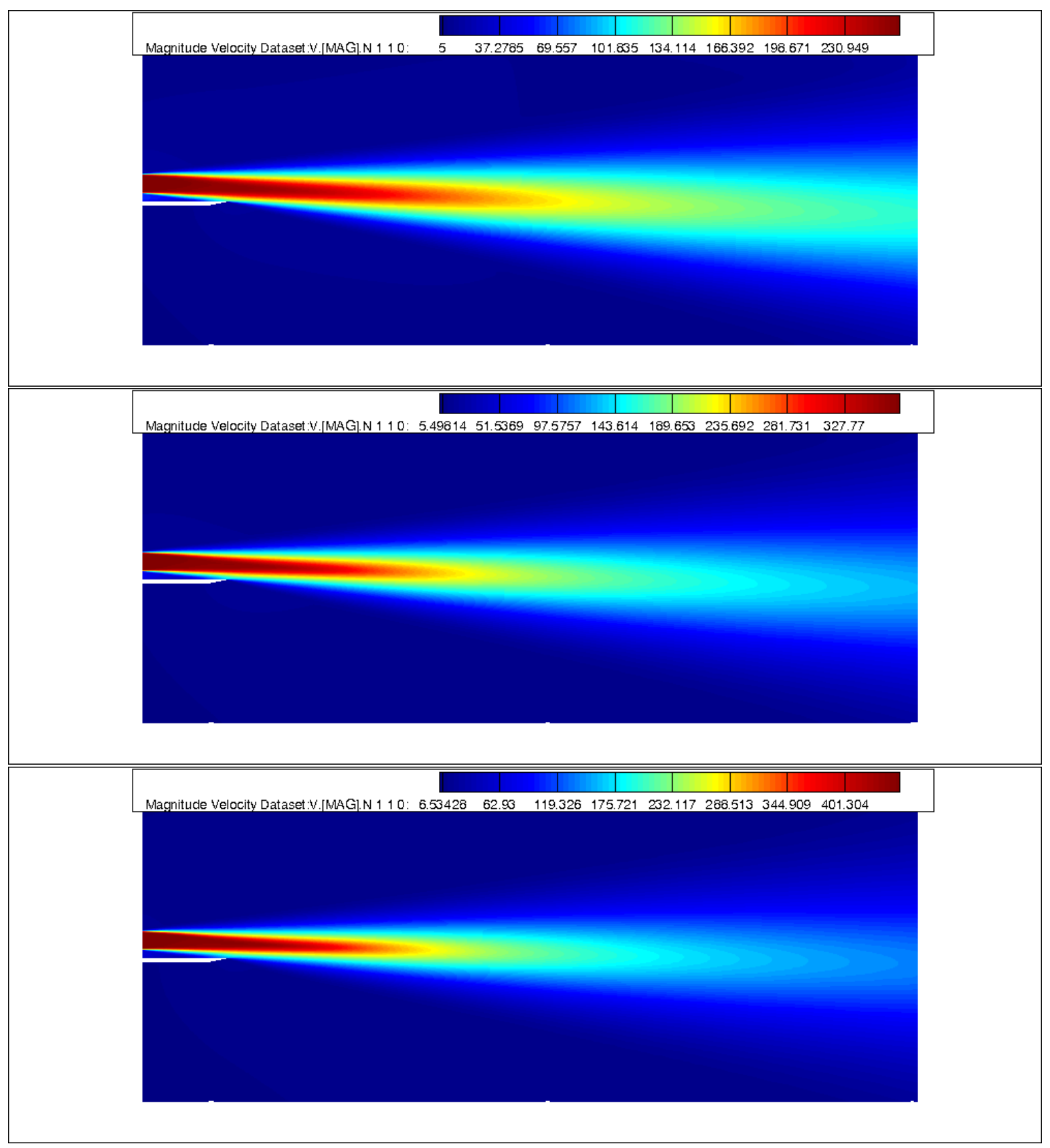

Figure 2: Mean velocity magnitude contours: a) $\mathrm{TR}=1$; b) $\mathrm{TR}=2$; c) $\mathrm{TR}=3$. 


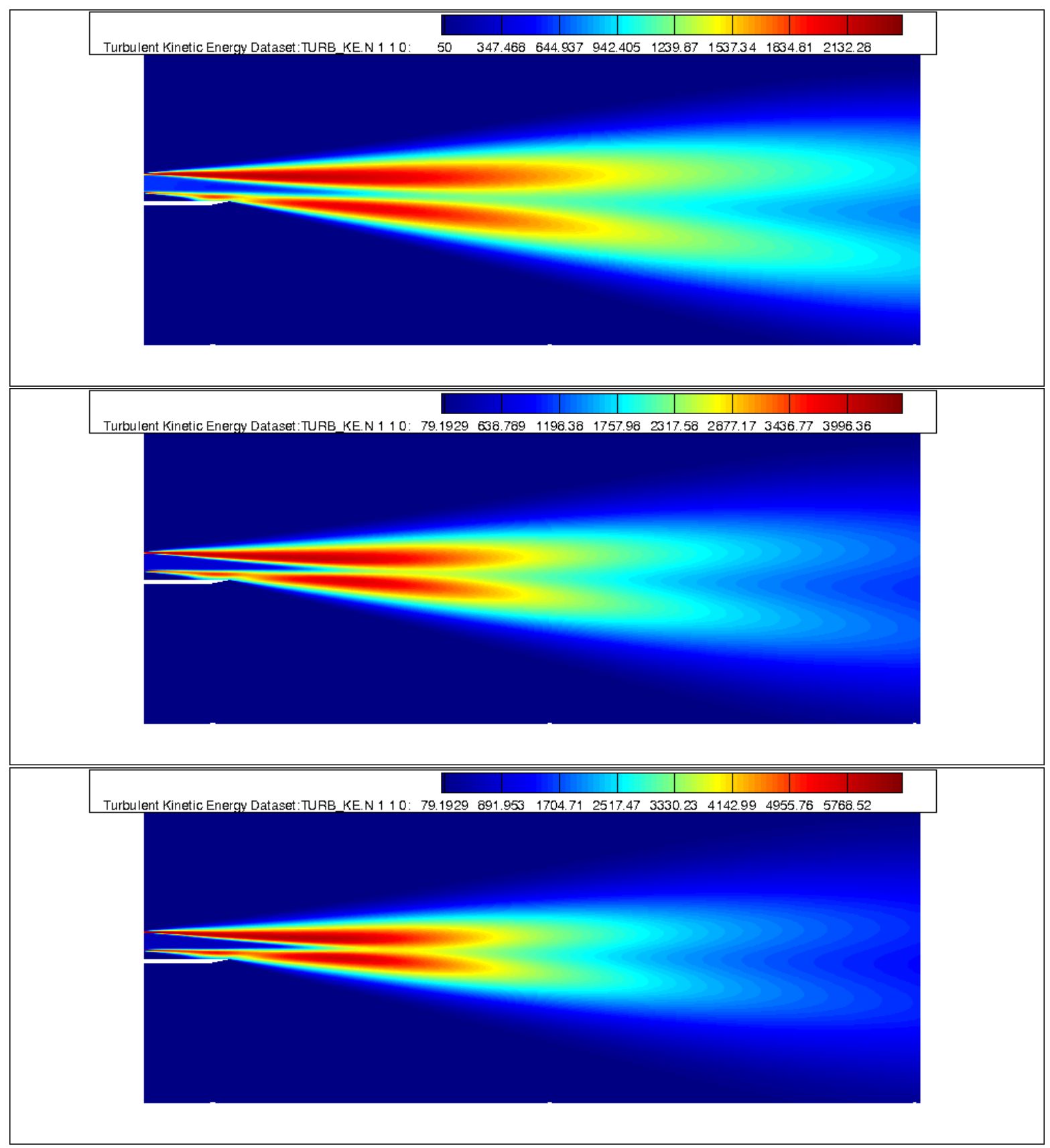

Figure 3: Turbulent kinetic energy contours: a) $\mathrm{TR}=1$; b) $\mathrm{TR}=2$; c) $\mathrm{TR}=3$.

Centerline mean velocity is plotted in figure 4 for all temperature ratios, showing that there is a slight decrease inside the potential core due to the presence of the flat surface (note that the nozzle exit is located in $x=-5$ ). Cross-flow profiles of mean velocity magnitude and turbulent kinetic energy in $x=-0.02 \mathrm{~m}$, which is in proximity to the trailing edge, inside the flat surface, and $x=0.1$ downstream of the trailing edge, are plotted in figures 5 and 7 , respectively. These profiles (especially the TKE profiles) indicate that the jet is significantly skewed by the presence of the flat surface. 


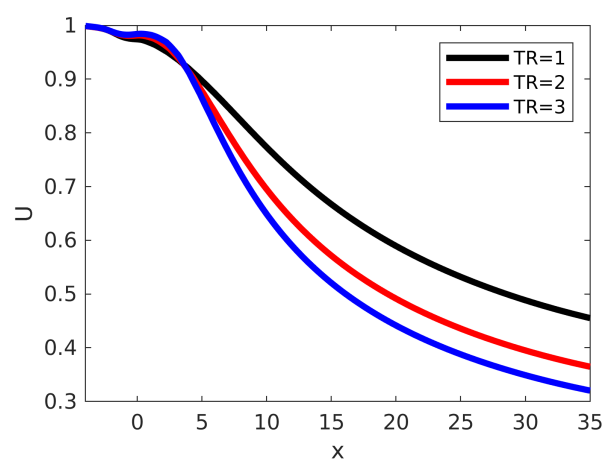

a)

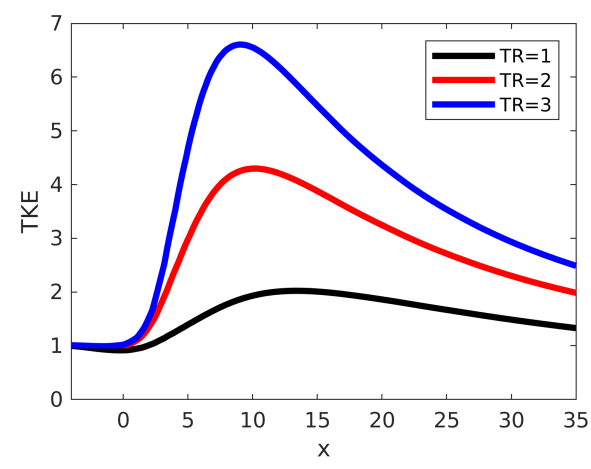

b)

Figure 4: Profiles along the jet centerline: a) Mean streamwise velocity; b) Turbulent kinetic energy.

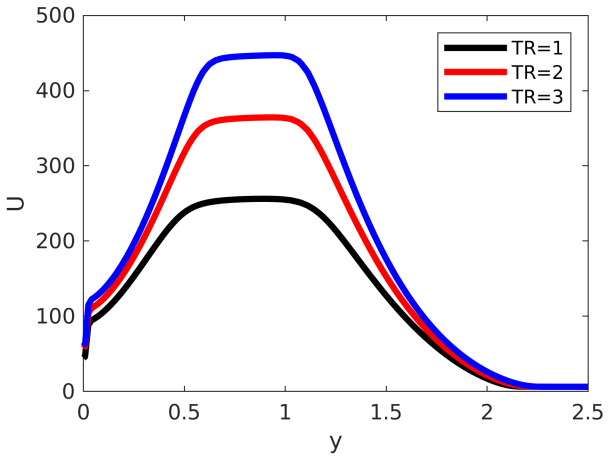

a)

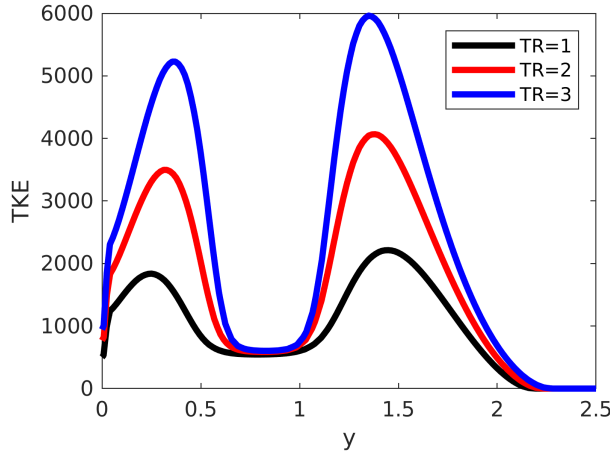

b)

Figure 5: Profiles along vertical coordinate $y$, at $x=-0.02 \mathrm{~m}$ (in proximity to the trailing edge): a) Mean streamwise velocity; b) Turbulent kinetic energy.

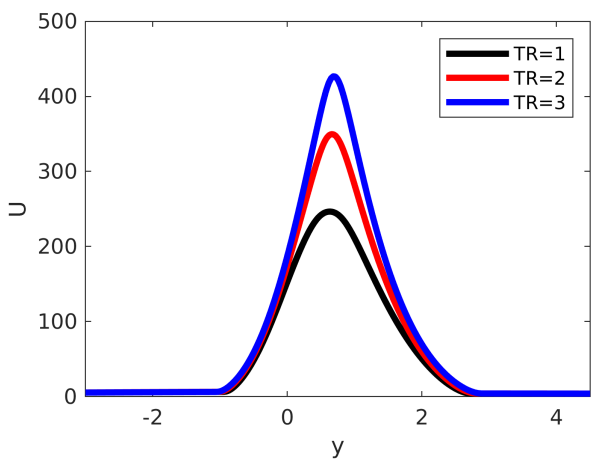

a)

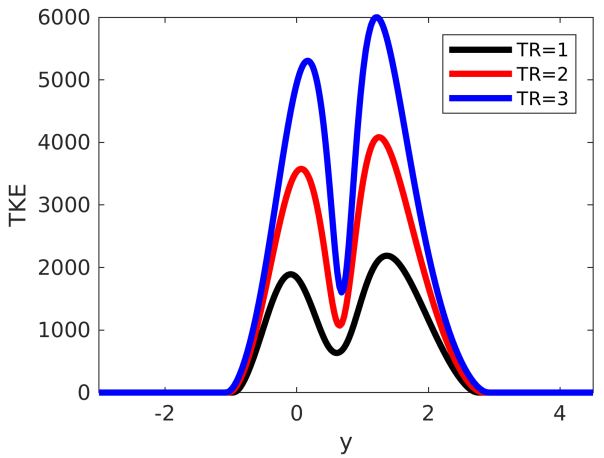

b)

Figure 6: Profiles along vertical coordinate $y$, at $x=0.1 \mathrm{~m}$ (downstream of the trailing edge): a) Mean streamwise velocity; b) Turbulent kinetic energy.

Finally, we show some preliminary results from LES for the temperature ratio $T R=1$. The mesh consists of approximately 10 millions grid points, clustered mostly in proximity to the jet region, while stretching is used in the farfield. A sponge layer is imposed at the outflow boundary to ensure that the fluctuations are gradually dissipated while they leave the flow domain. Iso-surfaces of Q-criterion $\left(Q=1 / 2\left[|\boldsymbol{\Omega}|^{2}-|\mathbf{S}|^{2}\right]\right.$, where $\mathbf{S}=1 / 2\left[\nabla \mathbf{v}+(\nabla \mathbf{v})^{T}\right]$ is the rate-of-strain tensor, and $\boldsymbol{\Omega}=1 / 2\left[\nabla \mathbf{v}-(\nabla \mathbf{v})^{T}\right]$ is the vorticity tensor) 
colored by the streamwise velocity component are shown in figure 7 . Acoustic waves radiating from the jet are plotted in gray contours (white lines are the gaps between blocks from the MPI decomposition).

a)

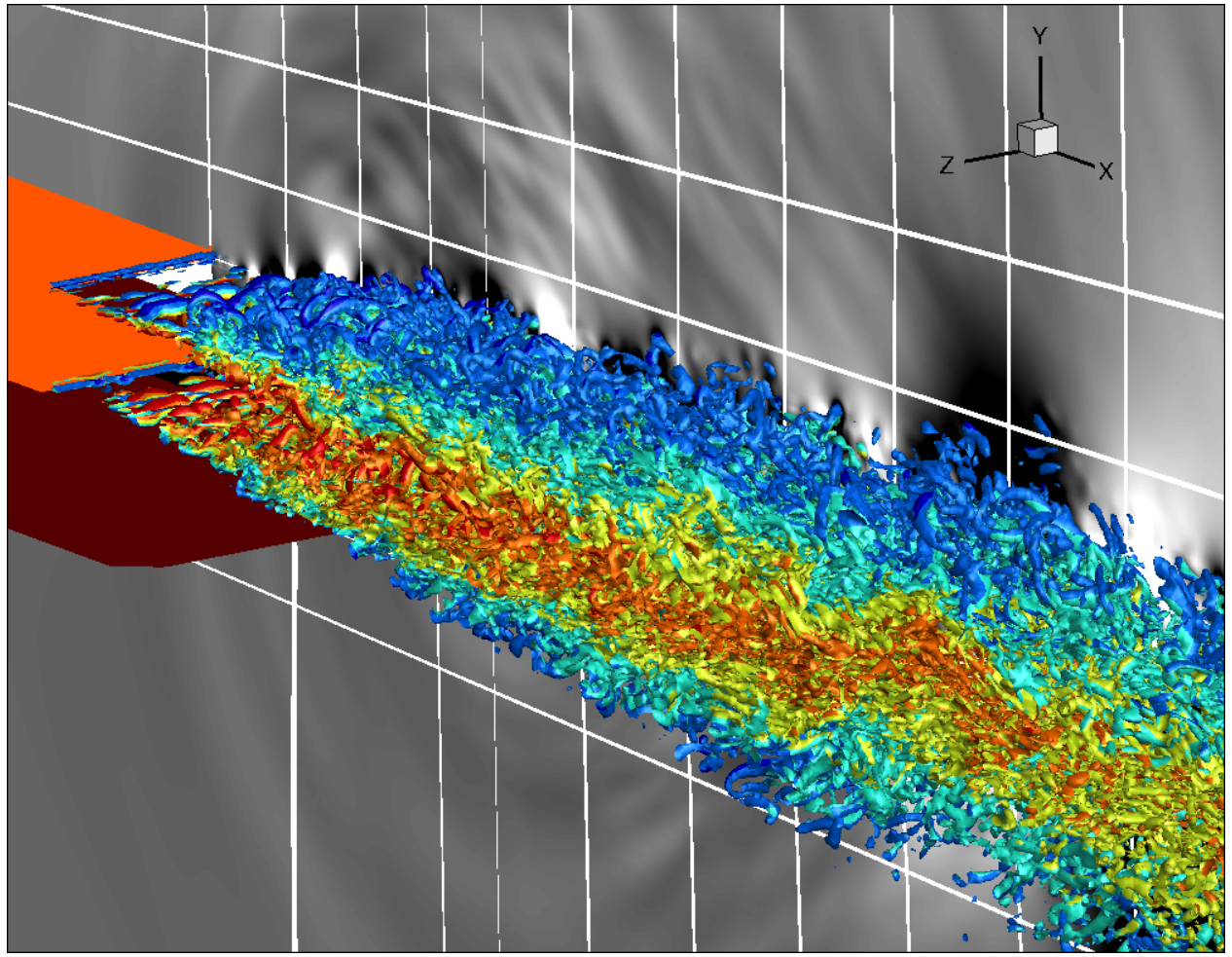

b)

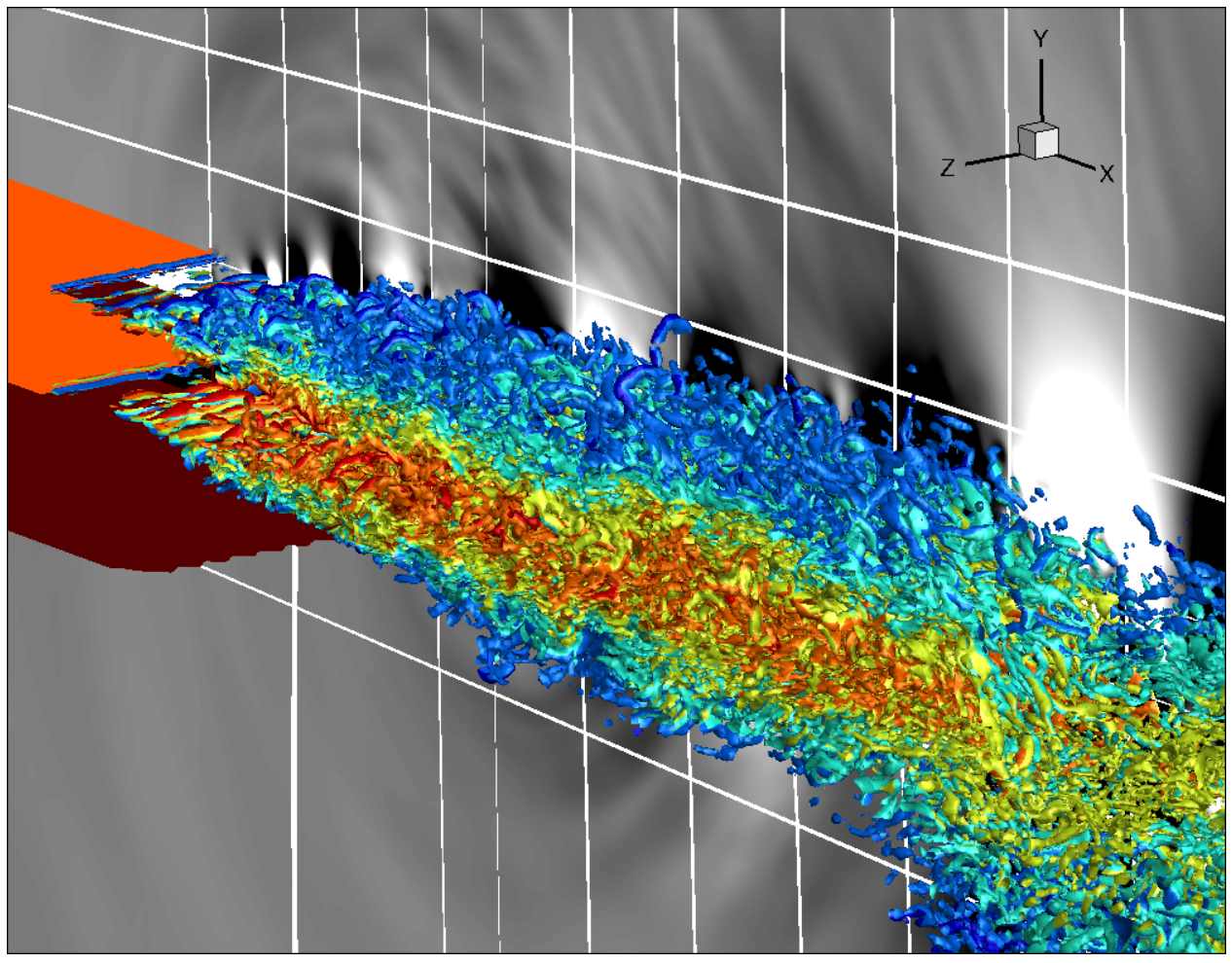

Figure 7: Iso-surfaces of Q-criterion colored by the streamwise velocity: a) Flat trailing edge; b) Deformations at the trailing edge.

In figure 8 , we show preliminary estimations of the noise radiation at three angles with respect to the 
jet axis. Jet surface interaction noise dominates at large polar angles (see Goldstein et al. ${ }^{8}$ for a study of AR8 nozzle and Afsar et al. ${ }^{1}$ where various rectangular nozzle jets were considered). Considering therefore the 90 degree spectrum in Fig. 8a, it is clear that the impact of surface deformations appears small at low frequencies. However, for St 1.0, the reduction (while highly oscillatory with frequency) fluctuates between $2-5 \mathrm{~dB}$ between $1.0<\mathrm{ST}<2.0$. For even larger Strouhal numbers the reduction grows to about 20dB, although the LES calculation may not be resolve the detailed structures of the turbulence at these frequencies. More analysis is therefore needed - this will be tackled in the final paper by an extensive parametric study in the terms of different geometrical and flow configurations. In addition, we will compare our predictions to the Rapid-distortion theory approach in Goldstein et al. ${ }^{8}$ by first computing the change in the power spectral density (i.e. total radiated sound minus jet noise) to isolate the effect of the edge on the acoustic spectrum.

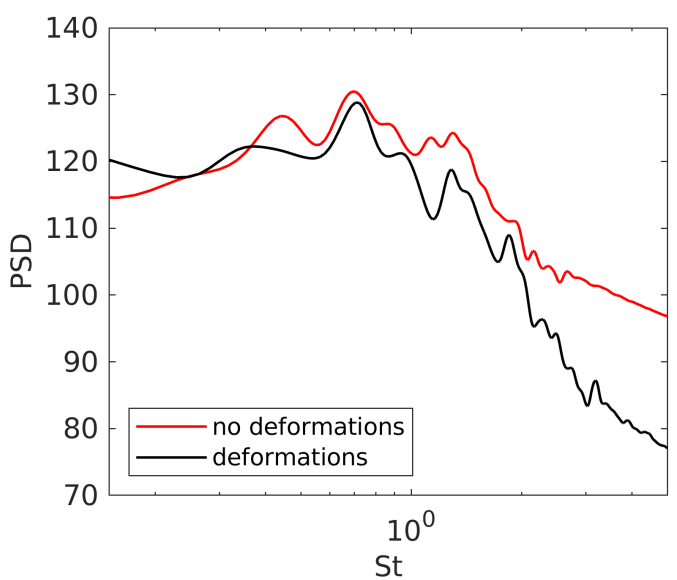

a)

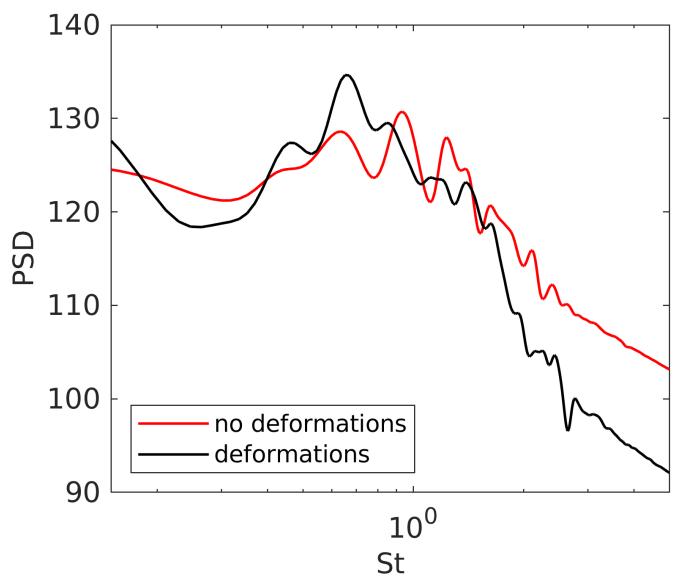

b)

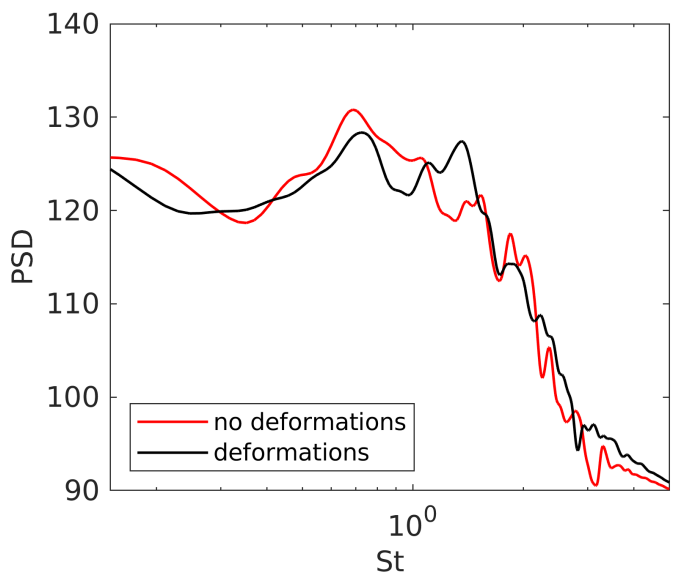

c)

Figure 8: Sound spectra in three probe locations: a) $\theta=90^{0}$; b) $\theta=75^{0}$; c) $\theta=60^{0}$.

\section{References}

\footnotetext{
${ }^{1}$ Afsar, M., Leib, S. \& Bozak, R., 6 Jan 2017, Effect of de-correlating turbulence on the low frequency decay of jet-surface interaction noise in sub-sonic unheated air jets using a CFD-based approach, In : Journal of Sound and Vibration. 386, p. $177 Đ 20730 \mathrm{p}$.

${ }^{2}$ Behrouzi, P. and McGuirk, J. J., Underexpanded Jet Development from a Rectangular Nozzle with Aft-deck, AIAA Journal, Vol. 53, No. 5, 2015, pp. 1287-1298.

${ }^{3}$ Bogey, C. and Bailly, C. (2004) A family of low dispersive and low dissipative explicit schemes for flow and noise computation, Journal of Computational Physics, Vol. 194, pp. 194-214.

${ }^{4}$ Bogey, C., Bailly, C., and Juve, D. (2002), A shock-capturing methodology based on adaptative spatial filtering for high-order non-linear computations, Journal of Computational Physics, Vol. 228, pp. 1447-1465.
} 
${ }^{5}$ Bridges, J. E., Noise from Aft Deck Exhaust Nozzle Differences in Experimental Embodiments, AIAA 2014-0876, 52th AIAA Aerospace Meeting, Maryland, USA, Jan 2014.

${ }^{6}$ Brown, C.A. , Jet-Surface Interaction Test: Far-Field Noise Results, J. Eng. Gas Turbines Power, 135(7), Jun. 2013.

${ }^{7}$ Brown, C. A. and Wernet, M.P. Jet surface interaction test: Flow measurements results, in 21st AIAA/CEAS Aeroacoustics Conference, Atlanta, GA, AIAA Paper 2014-3198, 2014.

${ }^{8}$ Goldstein, M. E., Leib, S. J. \& Afsar, M. Z., 30 Aug 2017, Generalized rapid-distortion theory on transversely sheared mean flows with physically realizable upstream boundary conditions: application to trailing edge problem, In : Journal of Fluid Mechanics. 824, p. 477-512 26 p.

${ }^{9}$ Ibrahim, M. K., Sawai, T., Obase, K., Mori, K. and Nakamura, Y., Experimental Investigation of Screech Tone Characteristics of Jet Interaction with a Flat Plate, AIAA Journal, Vol. 47, No. 9, 2009, pp. 2031-2038.

${ }^{10}$ Kennedy, C.A. and Carpenter, M.H. (1997) Comparison of several numerical methods for simulation of compressible shear layers, NASA Technical Report NASA-97-TP3484.

${ }^{11}$ Podboy, G., Jet-Surface Interaction Test: Phased Array Noise Source Localization Results, ASME GT2012-69801, 2012.

${ }^{12}$ Seiner, J. M. and Manning, J. C., Supersonic Jet Plume Interaction with a Flat Plate, Proceedings of the international Power Lift Conference, CA, USA, 1987.

${ }^{13} \mathrm{Shu}$, C.-W. and Osher, S. (1988) Efficient implementation of essentially non-oscillatory shock-capturing schemes, Journal of Computational Physics Vol. 77, No. 2, pp. 439-471.

${ }^{14}$ Tam, C.K.W. and Webb, J.C. (1993), Dispersion-relation-preserving finite difference schemes for Computational Aeroacoustics, Journal of Computational Physics, Vol. 107, pp. 262-281.

${ }^{15}$ Zaman, K.B.M.Q., Fagan, A. F., Clem, M. M. and Brown, C. A., Resonant Interaction of a Rectangular Jet with a Flat-plate, AIAA 2014-0877, 52th AIAA Aerospace Meeting, Maryland, Jan 2014.

${ }^{16}$ Zaman, K..B.M.Q., Brown, C. A. \& Bridges, J. E. 2013 Interaction of a rectangular jet with a flat-plate placed parallel to the flow. AIAA Paper 2013-2184. NASA/TM-2013-217879 (E-18684). 